\title{
The impact of food additives, artificial sweeteners and domestic hygiene products on the human gut microbiome and its fibre fermentation capacity
}

\author{
Konstantinos Gerasimidis ${ }^{1}$ (10 - Katie Bryden ${ }^{1} \cdot$ Xiufen Chen $^{1}$ - Eleftheria Papachristou ${ }^{1} \cdot$ Anais Verney $^{1}$. \\ Marine Roig ${ }^{1} \cdot$ Richard Hansen $^{2} \cdot$ Ben Nichols ${ }^{1} \cdot$ Rodanthi Papadopoulou $^{1} \cdot$ Alison Parrett $^{1}$
}

Received: 23 July 2019 / Accepted: 9 December 2019 / Published online: 18 December 2019

(C) The Author(s) 2019

\begin{abstract}
Purpose This study investigated the effect of food additives, artificial sweeteners and domestic hygiene products on the gut microbiome and fibre fermentation capacity.

Methods Faecal samples from 13 healthy volunteers were fermented in batch cultures with food additives (maltodextrin, carboxymethyl cellulose, polysorbate-80, carrageenan-kappa, cinnamaldehyde, sodium benzoate, sodium sulphite, titanium dioxide), sweeteners (aspartame-based sweetener, sucralose, stevia) and domestic hygiene products (toothpaste and dishwashing detergent). Short-chain fatty acid production was measured with gas chromatography. Microbiome composition was characterised with 16S rRNA sequencing and quantitative polymerase chain reaction (qPCR).

Results Acetic acid increased in the presence of maltodextrin and the aspartame-based sweetener and decreased with dishwashing detergent or sodium sulphite. Propionic acid increased with maltodextrin, aspartame-based sweetener, sodium sulphite and polysorbate- 80 and butyrate decreased dramatically with cinnamaldehyde and dishwashing detergent. Branchedchain fatty acids decreased with maltodextrin, aspartame-based sweetener, cinnamaldehyde, sodium benzoate and dishwashing detergent. Microbiome Shannon $\alpha$-diversity increased with stevia and decreased with dishwashing detergent and cinnamaldehyde. Sucralose, cinnamaldehyde, titanium dioxide, polysorbate-80 and dishwashing detergent shifted microbiome community structure; the effects were most profound with dishwashing detergent $\left(R^{2}=43.9 \%, p=0.008\right)$ followed by cinnamaldehyde $\left(R^{2}=12.8 \%, p=0.016\right)$. Addition of dishwashing detergent and cinnamaldehyde increased the abundance of operational taxonomic unit (OTUs) belonging to Escherichia/Shigella and Klebsiella and decreased members of Firmicutes, including OTUs of Faecalibacterium and Subdoligranulum. Addition of sucralose and carrageenan-kappa also increased the abundance of Escherichia/Shigella and sucralose, sodium sulphite and polysorbate-80 did likewise to Bilophila. Polysorbate-80 decreased the abundance of OTUs of Faecalibacterium and Subdoligranulum. Similar effects were observed with the concentration of major bacterial groups using qPCR. In addition, maltodextrin, aspartame-based sweetener and sodium benzoate promoted the growth of Bifidobacterium whereas sodium sulphite, carrageenan-kappa, polysorbate- 80 and dishwashing detergent had an inhibitory effect.

Conclusions This study improves understanding of how additives might affect the gut microbiota composition and its fibre metabolic activity with many possible implications for human health.
\end{abstract}

Keywords Food additives $\cdot$ Microbiota $\cdot$ Fermentation capacity $\cdot$ Fibre $\cdot$ Gut microbiome

\section{Introduction}

Electronic supplementary material The online version of this article (https://doi.org/10.1007/s00394-019-02161-8) contains supplementary material, which is available to authorized users.

Konstantinos Gerasimidis

konstantinos.gerasimidis@glasgow.ac.uk

Extended author information available on the last page of the article
A great amount of research has investigated the role of dietary nutrients, or dietary patterns in general, on the gut microbiome. Dietary fibre has attracted the most interest, mainly due to the inability of the human body to utilise it, and the capability of the gut microbiome to ferment it using a broad spectrum of enzymes not encoded in the human 
genome cannot encode [1]. Short-chain fatty acids (SCFA) are the end-product of fibre fermentation and the SCFA produced are dependent on the host's diet and microbiome composition. Species within Bacteroides produce primarily acetic acid and propionic acid [2, 3]; members of Clostridium leptum cluster produce butyric acid from fibre fermentation and Bifidobacterium produces lactate and acetic acid from carbohydrate fermentation [4]. The branch chain fatty acids (BCFA) iso-butyric acid and iso-valeric acid are produced from protein breakdown, particularly in the absence of fermentable carbohydrate. Yet, the human gut microenvironment dynamics are more complex and characterised by an extensive degree of inter-species synergy and crossfeeding. It is, therefore, important to study the interactions between diet and the gut microbiome in the context of the entire microbial community and not as microbes in isolation. SCFA are critical bacterial products involved, not only locally in gut health, but in whole-body homeostasis. Along with an increased microbial diversity, high butyric acid concentration in the gut has been used as an indicator of healthy status of the microbiome. In contrast, reduced diversity, low luminal production of SCFA and dysbiosis have been proposed as primary events of inflammatory bowel disease, diabetes and obesity [5-8].

Our diet has evolved enormously and rapidly over the last century, in parallel with food preservation and processing and increased use of industrialised and domestic hygiene products. While food industrialisation has protected humanity from infectious diseases, the secondary effect this may have on gut microbiome-dependent host health, and the net impact on the incidence of non-communicable diseases has only relatively recently been considered. A Mediterranean diet with increased consumption of legumes, cereals, fruit and vegetables, and its health-promoting effects, influences the gut microbiome [9]. The Western diet, which includes food additives and preservatives, has contrastingly been associated with non-communicable diseases [10]. Food additives and artificial sweeteners have become increasingly prevalent within our diet, with more than $50 \%$ of available food in UK households being ultra-processed [11]. While food additives are evaluated rigorously for their effects on the host, health testing of food additives fails to include their effect on the human gut microbiome and by proxy long-term host health [12]. Recent studies in animals have indicated that food additives can have adverse effects on colonic and cardiovascular health, mediated by the gut microbiome and changes in the gut mucus layer. It has been shown that food emulsifiers, such as polysorbates and carboxymethyl cellulose can increase intestinal permeability, alter microbiota composition, promote Escherichia coli translocation across the epithelium and in $\mathrm{M}$ cells in-vitro causing gut inflammation $[10,13]$. Likewise, the body of evidence on artificial sweeteners indicates that there are adverse metabolic outcomes in rodents owing to the onset of microbial dysbiosis [14-16]. Cumulative ingestion of residual products from regular use of domestic hygiene products may influence the human gut microbiome and, by extension, the health of the host. In epidemiological research, increased use of dishwashers, which reduce residual domestic detergent on dishware and consequent accidental ingestion, was associated with a decrease in cardiovascular disease [17]. Although for some food additives, artificial sweeteners and domestic hygiene products a large amount will be digested or degraded in the upper part of the gastrointestinal tract, residual amounts can still reach the colon. Others, like carrageenans and carboxylmethyl cellulose will reach the colon in similar amounts to those ingested.

It is, therefore, important to study the effect of food additives, artificial sweeteners and domestic hygiene products may have on gut microbiota composition and its fibre fermentation capacity, the most important bacterial function for host health. There is currently limited knowledge on the effect of additives on the human gut microbiota, and research to date has predominantly occurred in animal models with a paucity of evidence in humans. This preclinical study investigated the effect that commonly consumed food additives, including emulsifiers, artificial sweeteners and domestic hygiene products might have on the healthy human microbiota composition and its fibre fermentation capacity using in-vitro batch faecal fermentations.

\section{Subjects and methods}

\section{Participants}

Thirteen young healthy adults (females, $n=7$; mean, (SD); age: $24.8,(2.2)$ years; body mass index (BMI) 21.9, (2.8) $\mathrm{kg} / \mathrm{m}^{2}$ ) donated a single faecal sample. Participants who had used antibiotics within the three months prior were not eligible to participate. Participants provided informed consent. The study received ethical approval by the Medical, Veterinary and Life Sciences Research Ethics Committee, at the University of Glasgow.

\section{In-vitro batch faecal fermentation studies}

Faecal samples were collected in disposable containers and processed within one hour of defecation. From each donor, a faecal slurry $(16 \% \mathrm{w} / \mathrm{v})$ was prepared using $16 \mathrm{~g}$ of faecal matter homogenised in $100 \mathrm{ml}$ Sorensen's buffer $\mathrm{pH} 7$, boiled and degassed under oxygen-free nitrogen stream. The faecal slurry was strained through 30-denier nylon stockings to remove coarse material and remained in suspension by continuous agitation using a magnetic stirrer. In a $150 \mathrm{ml}$ flask, $5 \mathrm{ml}$ of $16 \%$ faecal slurry were added along with $42 \mathrm{ml}$ 
of in-house prepared fermentation medium, $2 \mathrm{ml}$ of reducing solution, $400 \mathrm{mg}$ of fibre substrate (see below) and one of the additives in testing. Assuming that an average person has a faecal output of $120 \mathrm{~g} /$ day [18] and a recommended intake of the fibre of $30 \mathrm{~g} /$ day, this would be equivalent to roughly double the amount of fibre available for fermentation per $g$ of faeces.

The fermentation medium was prepared in-house (1 litre). It consisted of $225 \mathrm{ml}$ of macromineral solution $(0.04 \mathrm{M}$ $\mathrm{Na}_{2} \mathrm{HPO}_{4}, 0.046 \mathrm{M} \mathrm{KH}_{2} \mathrm{PO}_{4}, 0.002 \mathrm{M} \mathrm{MgSO}_{4} \cdot 7 \mathrm{H}_{2} \mathrm{O}$ ), $225 \mathrm{ml}$ buffer solution $\left(0.051 \mathrm{M} \mathrm{NH}_{4} \mathrm{HCO}_{3}\right.$ and $0.417 \mathrm{M}$ $\left.\mathrm{NaHCO}_{3}\right), 112.5 \mu \mathrm{l}$ of micromineral solution $(0.898 \mathrm{M}$ $\mathrm{CaCl}_{2} \cdot 2 \mathrm{H}_{2} \mathrm{O}, 0.505 \mathrm{M} \mathrm{MnCl}_{2} \cdot 4 \mathrm{H}_{2} \mathrm{O}, 0.042 \mathrm{M} \mathrm{CoCl}_{2} \cdot 6 \mathrm{H}_{2} \mathrm{O}$, and $\left.0.296 \mathrm{M} \mathrm{FeCl}_{3} \cdot 6 \mathrm{H}_{2} \mathrm{O}\right), 1.125 \mathrm{ml}$ of $0.1 \%$ resazurin solution, $450 \mathrm{ml}$ of $5 \mathrm{mg} / \mathrm{mL}$ Tryptone, $100 \mathrm{mg}$ of mucin from porcine stomach, and $76 \mathrm{mg}$ of mixed bile extract from porcine. Once the solution was made, it was boiled, degassed under oxygen-free nitrogen, and adjusted to $\mathrm{pH} 7$ to mimic the distal intestinal environment. Reducing solution $(50 \mathrm{ml})$ was made up of $2 \mathrm{ml}$ of $1 \mathrm{M} \mathrm{NaOH}, 312.5 \mathrm{mg}$ of cysteine hydrochloride and $312.5 \mathrm{mg}$ of $\mathrm{Na}_{2} \mathrm{~S} \cdot 9 \mathrm{H}_{2} \mathrm{O}$.

The fibre substrate was made up of $100 \mathrm{mg}$ of apple pectin (SIGMA, Pectin, from apple), $100 \mathrm{mg}$ of raftilose (Beneo $^{\mathrm{TM}}$, Orafti P95), $100 \mathrm{mg}$ of $\alpha$-cellulose (SIGMA $^{\mathrm{TM}}$, $\alpha$-CELLULOSE), and $100 \mathrm{mg}$ of high resistant maize starch (National StarchTM, HI-MAIZE[TM] 260). We chose these fibres as indicative of food consumed in the UK diet [19].

Eight food additives [maltodextrin, carboxymethyl cellulose, polysorbate-80, carrageenan-kappa, sodium benzoate, sodium sulphite, titanium dioxide, cinnamaldehyde], three artificial sweeteners [aspartame-based sweetener, sucralose, stevia], and two domestic hygiene products [toothpaste, dishwashing detergent] were used. Test amounts were based on the acceptable daily intake or estimated daily consumption, assuming an average male adult weighing $75 \mathrm{~kg}$ (Online Resource 1). Where the estimated daily consumption was relatively large (maltodextrin, carboxymethyl cellulose, polysorbate-80, carrageenan-kappa, aspartamebased sweetener), the amount tested was standardised to $500 \mathrm{mg}$. Likewise, where estimated daily consumption was relatively small (stevia, cinnamaldehyde, sodium benzoate, sodium sulphite, sucralose), the amount tested was $50 \%$ of the acceptable daily intake. For the toothpaste and the dishwashing detergent, the amount tested was $100 \%$ of estimated accidental intake (Online Resource 1). Selection of additives was based on previous research which implicated them in the onset of non-communicable diseases including inflammatory bowel disease and metabolic syndrome [10, 20, 21].

Thirteen fermentation flasks, one for each of the additives above, and a non-additive blank (hereafter referred to as control) were degassed under oxygen-free nitrogen stream and incubated in a shaking water bath at $37{ }^{\circ} \mathrm{C}$ at 60 strokes/min for $24 \mathrm{~h}$. A baseline sample was collected from the control prior to incubation start and from all other additives and the control after $24 \mathrm{~h}$ of incubation. Aliquots of fermentation slurry for SCFA analysis were collected and stored in 3:1 ratio with $1 \mathrm{M} \mathrm{NaOH}$ at $-20{ }^{\circ} \mathrm{C}$ until analysis. Fermentation slurry aliquots were stored at $-80^{\circ} \mathrm{C}$ and total DNA was extracted within a month of collection.

\section{Measurement of net SCFA production}

The SCFA (acetic acid, propionic acid, butyric acid, valeric acid, caproic acid, heptanoic acid, and caprylic acid) and BCFA (iso-butyric acid and iso-valeric acid) were extracted from acidified slurries three times in total using diethyl ether. Extracts were analysed using Gas Chromatography (Agilent 7890A) with flame ionisation detector, as described previously $[22,23]$. Each of the SCFA was quantified against calibration curves plotted using authentic external standards [acetic acid $(185.8 \mathrm{mM})$, propionic acid $(144.5 \mathrm{mM})$, butyric acid $(114.2 \mathrm{mM})$, valeric acid $(83.4 \mathrm{mM})$, caproic acid $(52.6 \mathrm{mM})$, heptanoic acid $(65.8 \mathrm{mM})$, caprylic acid $(53.2 \mathrm{mM})$, isobutyric acid $(97.3 \mathrm{mM})$, and isovaleric acid $(87.0 \mathrm{mM})$ all stored in $2 \mathrm{M} \mathrm{NaOH}$ and using 2-ethylbutyric acid $(74.0 \mathrm{mM})$ as internal standard. All samples from the same participant were analysed in the same run to minimise inter-assay variation. Each sample was measured twice, and in all cases the average concentration was calculated unless the $\%$ co-efficient of variation was greater than $10 \%$ in which case a third replicate was analysed. Concentration of SCFA $(\mu \mathrm{mol})$ is reported per volume $(\mathrm{ml})$ of fermentation slurry.

\section{Extraction of genomic DNA from fermentation slurries}

In a subset of 8 participants, 16S rRNA amplicon sequencing of the human gut microbiome and quantification of total and 5 dominant bacterial groups were performed. Samples were thawed at room temperature and after centrifugation at $12,000 \mathrm{~g}$ for $5 \mathrm{~min}$, genomic DNA from the resultant pellet was extracted using the DNeasy Powersoil Kit. The purity and concentration of extracted DNA was quantified using the NanoDrop ${ }^{\mathrm{TM}} 1000$ and Qubit.

\section{Quantification of dominant bacterial groups of the human gut microbiome}

Quantitative PCR (qPCR) was performed using TaqMan ${ }^{\mathrm{TM}}$ chemistry and quantified against serial dilution of standards prepared from pure bacterial cultures as described previously [22]. Total bacteria and 5 different bacterial groups were targeted (Bacteroides/Prevotella, Bifidobacterium, Blautia coccoides, Clostridium leptum and E. coli) (Online Resource 2). The PCR reaction consisted of $7.5 \mu \mathrm{l} \mathrm{Taqman}{ }^{\mathrm{TM}}$ gene expression master mix, $2.25 \mu \mathrm{l}$ nuclease-free water, 
$0.5 \mu \mathrm{l}$ bovine serum albumin, $1.5 \mu \mathrm{l}$ forward primer $(9 \mu \mathrm{M})$, $1.5 \mu \mathrm{l}$ reverse primer $(9 \mu \mathrm{M})$ and $0.75 \mu \mathrm{l}$ probe $(2.5 \mu \mathrm{M})$. qPCR was performed in triplicates and averages calculated for replicates where $\mathrm{Ct}$ difference was less than $0.2 \mathrm{Ct}$.

\section{Characterisation of global microbiome with 165 rRNA sequencing}

Sequencing of the V4 region of the 16S rRNA gene was performed on the MiSeq (Illumina, Essex, UK) platform using $2 \times 250$ bp paired-end reads [23, 24].

\section{Bioinformatics}

To enable analysis of the gut microbiome, $97 \%$ operational taxonomic units (OTUs) were generated from the 16S rRNA sequences using an adaptation of the VSEARCH pipeline (https://github.com/torognes/vsearch/wiki/VSEARCH-pipel ine) [25]. Quality filtering was performed on the combined paired reads with a maximum allowed expected error rate of 0.5 base pairs per read. Sequences longer than $275 \mathrm{bp}$ and shorter than $225 \mathrm{bp}$ were also filtered out. The next steps involved dereplication, removal of singleton sequences and preclustering at $98 \%$. Chimeras were removed using the VSEARCH implementation of the UCHIME de-novo algorithm followed by the UCHIME reference-based method in conjunction with the 'Gold' ChimeraSlayer reference dataset $[26,27]$. Finally, OTUs were assigned by clustering the remaining sequences at $97 \%$ and taxonomically classified using a naive Bayesian classifier method implemented in the dada2 $\mathrm{R}$ package [28].

\section{Statistical analysis}

Data are presented as medians and interquartile (Q1-Q3) range. One-sample Wilcoxon (non-normally distributed data) or paired $t$ test (normally distributed data) was used to calculate the difference between each additive and the control. Microbiome analysis using the 16S rRNA gene sequences was carried out in $\mathrm{R}$ version 3.5.3. The alpha diversity measures (i.e. rarefied richness, Chao1 richness estimate, Shannon diversity index, and Pielou's evenness) were all calculated using the vegan package [29]. Permutation ANOVA results were also generated using vegan on both Bray-Curtis and UniFrac distance matrices. In the case of UniFrac the phylogenetic tree was generated using FastTree 2 [30]. Nonmetric multidimensional scaling (NMDS) was performed with the phyloseq package [31] and was used to visualise overall community structure in the form of ordination plots. Differentially abundant taxa were found using paired t-tests on log-relative abundances. Only significant differences greater than $0.5 \log$ s are reported. Significance was set at 0.05 .

\section{Results}

\section{Effect of additives on net SCFA production}

Figure 1 displays the net production of SCFA and Table 1 the median of the difference in their concentration with respect to the $24 \mathrm{~h}$ control, for each additive. Fermentation of the control for $24 \mathrm{~h}$ increased the production of total SCFA ( 0 h vs $24 \mathrm{~h} ; 1.73$ vs 45.36 , $\mu \mathrm{mol} / \mathrm{ml} ; p<0.0001$ ) (Fig. 1). Addition of maltodextrin and aspartame-based sweetener produced the highest median concentration of total SCFA whereas the dishwashing detergent the lowest (Fig. 1). Considering the individual SCFA, maltodextrin $(p<0.001)$ and aspartame-based sweetener $(p<0.001)$ increased the production of acetic acid whilst in contrast, dishwashing detergent $(p<0.001)$ and sodium sulphite $(p=0.036)$ caused a significant decrease in acetic acid production compared with the control (Fig. 1). Production of propionic acid was increased when maltodextrin $(p=0.014)$, polysorbate-80 $(p=0.044)$, sodium sulphite $(p=0.011)$ or the aspartame-based sweetener $(p=0.034)$ were present (Fig. 1). Addition of cinnamaldehyde $(p=0.006)$ or dishwashing detergent $(p=0.012)$ significantly decreased the production of butyric acid when compared with the control; a similar non-significant effect $(p=0.052)$ was also observed for sodium sulphite (Fig. 1). Compared with the control, sucralose $(p=0.025)$ and polysorbate- 80 $(p=0.003)$ significantly increased production of valeric acid whereas when maltodextrin $(p=0.002)$, cinnamaldehyde $(0.014)$, aspartame-based sweetener $(p=0.002)$ and the dishwashing detergent $(p=0.002)$ were added a significant decrease was observed (Fig. 1). There was a significant decrease in the production of caproic acid when maltodextrin $(p=0.012)$, cinnamaldehyde $(p=0.021)$, sodium sulphite $(p=0.014)$, aspartame-based sweetener $(p=0.002)$ or dishwashing detergent $(p=0.010)$ were added (Fig. 1). Caprylic acid significantly increased in the presence of maltodextrin $(p=0.006)$, polysorbate- 80 $(p=0.002)$, aspartame-based sweetener $(p=0.009)$ and dishwashing detergent (0.035) (Fig. 1). With regard to the BCFA, there was a significant decrease in the production of isobutyric acid when maltodextrin $(p=0.002)$, cinnamaldehyde $(p=0.025)$, sodium butyrate $(p=0.014)$, aspartame-based sweetener $(p<0.001)$ or dishwashing detergent $(p=0.002)$ were added (Fig. 1). Similar effects were also seen for isovaleric acid [maltodextrin $(p=0.002)$, cinnamaldehyde $(p=0.041)$, sodium benzoate $(p=0.004)$, aspartame-based sweetener $(p<0.001)$ and dishwashing detergent $(p=0.002)$ ] (Fig. 1). Carboxymethyl cellulose, toothpaste, carrageenan-kappa, titanium dioxide, sodium benzoate and stevia had no effect on the 

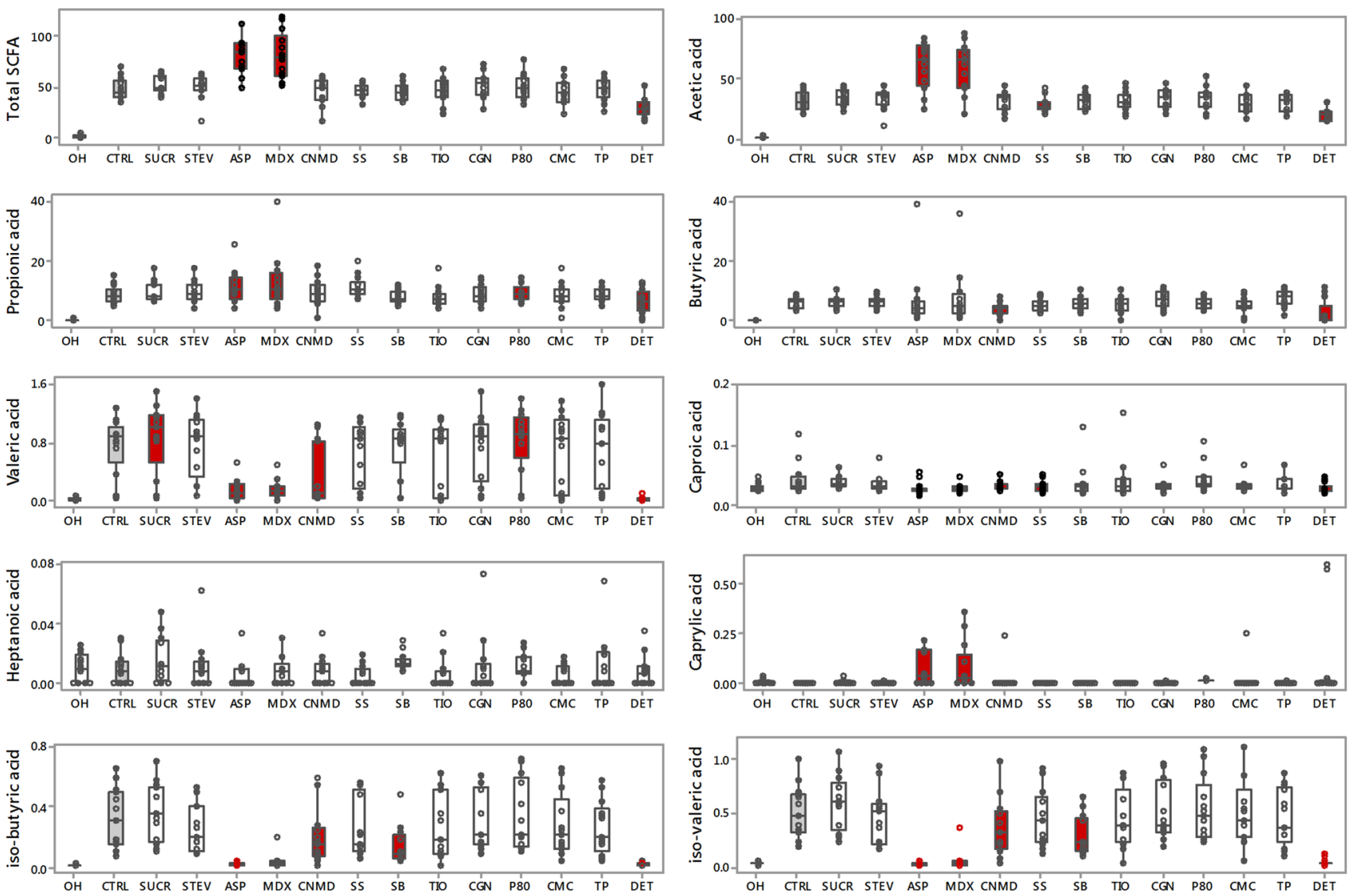

Fig. 1 Baseline and net production of total and individual short chain fatty acids $(\mu \mathrm{mol} / \mathrm{ml})$ following $24 \mathrm{~h}$ batch faecal fermentation of fibre with food additives, artificial sweeteners and domestic hygiene products. Red filling boxplot indicates significant difference $(p<0.05)$ compared with the CTRL (displayed with grey filling box-

production of any SCFA or BCFA. The effect of each of the substrates on the proportional ratio or SCFA are displayed in Online Resource 3.

\section{Effect of additives on microbiome diversity indices}

Compared to the control group, the addition of dishwashing detergent significantly decreased all metrics of microbiome $\alpha$-diversity, including OTU richness, evenness and the Shannon diversity index (Fig. 2). Incubation of faecal microbiota with cinnamaldehyde decreased the Shannon diversity index whereas an effect in the opposite direction was provoked by stevia. The effects of stevia and cinnamaldehyde on Shannon diversity index were due to an effect on microbiome community evenness rather than an impact on OTU richness (Fig. 2). There were no other significant effects on $\alpha$-diversity indices for the rest of the substrates. plot); $O H$ baseline, $C T R L$ control, $S U C R$ sucralose, $S T E V$ stevia, ASP aspartame based sweetener, $M D X$ maltodextrin, $C N M D$ cinnamaldehyde, $S S$ sodium sulphite, $S B$ sodium benzoate, $T I O$ titanium dioxide, $C G N$ carrageenan-kappa, $P 80$ polysorbate-80, CMC carboxymethyl cellulose, TP toothpaste, DET detergent

\section{Effect of additives on microbiome community structure}

Addition of sucralose, cinnamaldehyde, titanium dioxide, polysorbate- 80 and dishwashing detergent induced significant shifts in microbiome community structure ( $\beta$-diversity) using the Bray-Curtis dissimilarity index (Fig. 3). The most pronounced effects were from dishwashing detergent followed by cinnamaldehyde, which explained $43.9 \%(p=0.008)$ and $12.8 \%(p=0.016)$ of the variance in microbiome community structure, respectively. The effects of sucralose $\left(R^{2}=5.6 \%, p=0.023\right)$, polysorbate- $80\left(R^{2}=3.6 \%, p=0.023\right)$ and titanium dioxide $\left(R^{2}=4.5 \%, p=0.023\right)$ were significant but less pronounced. When we looked at the effects of food additives, artificial sweeteners and domestic hygiene products on their microbiome community structure using UniFrac distances, which consider OTU phylogenetic relatedness, a significant effect was observed for cinnamaldehyde 


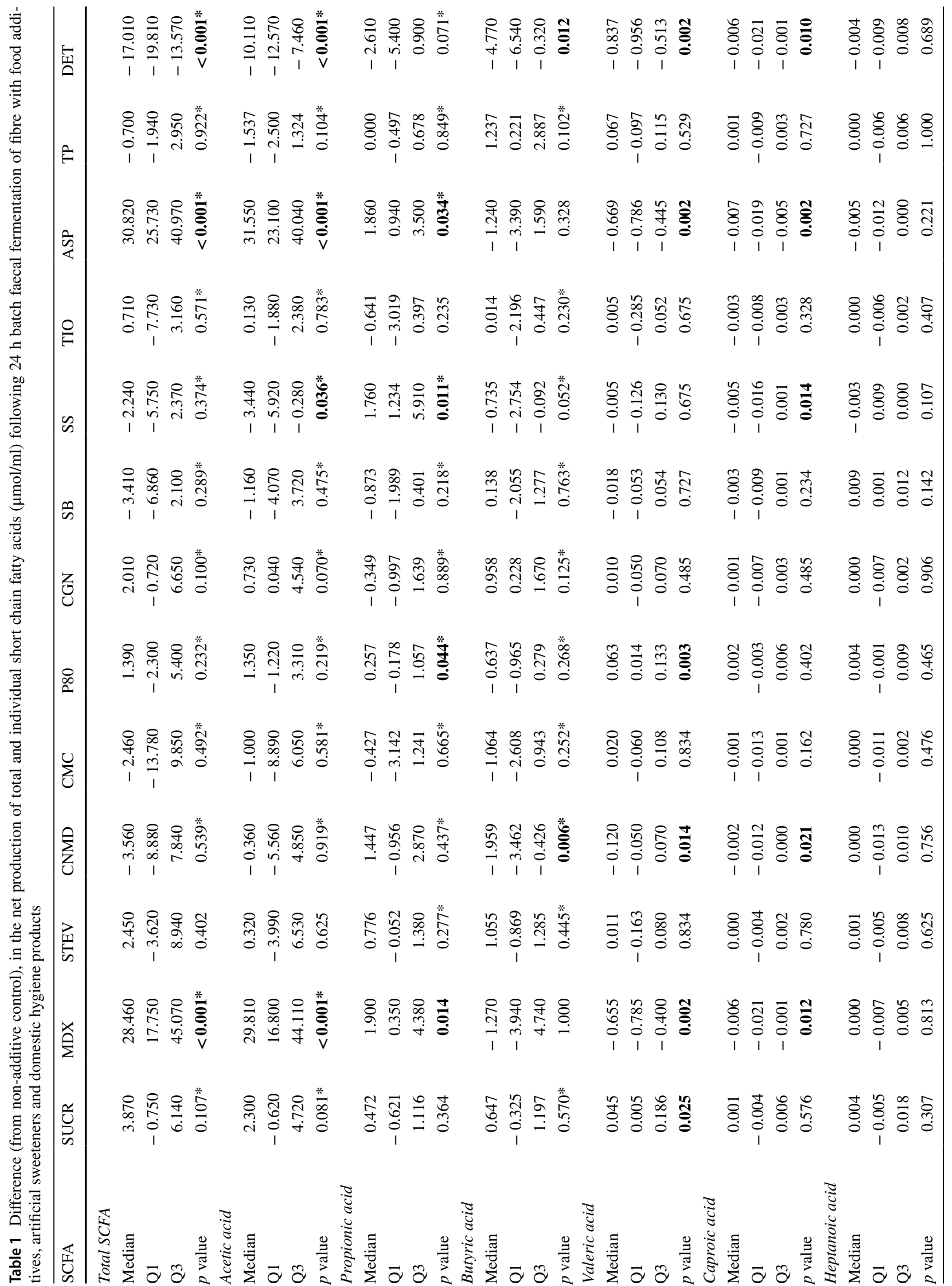




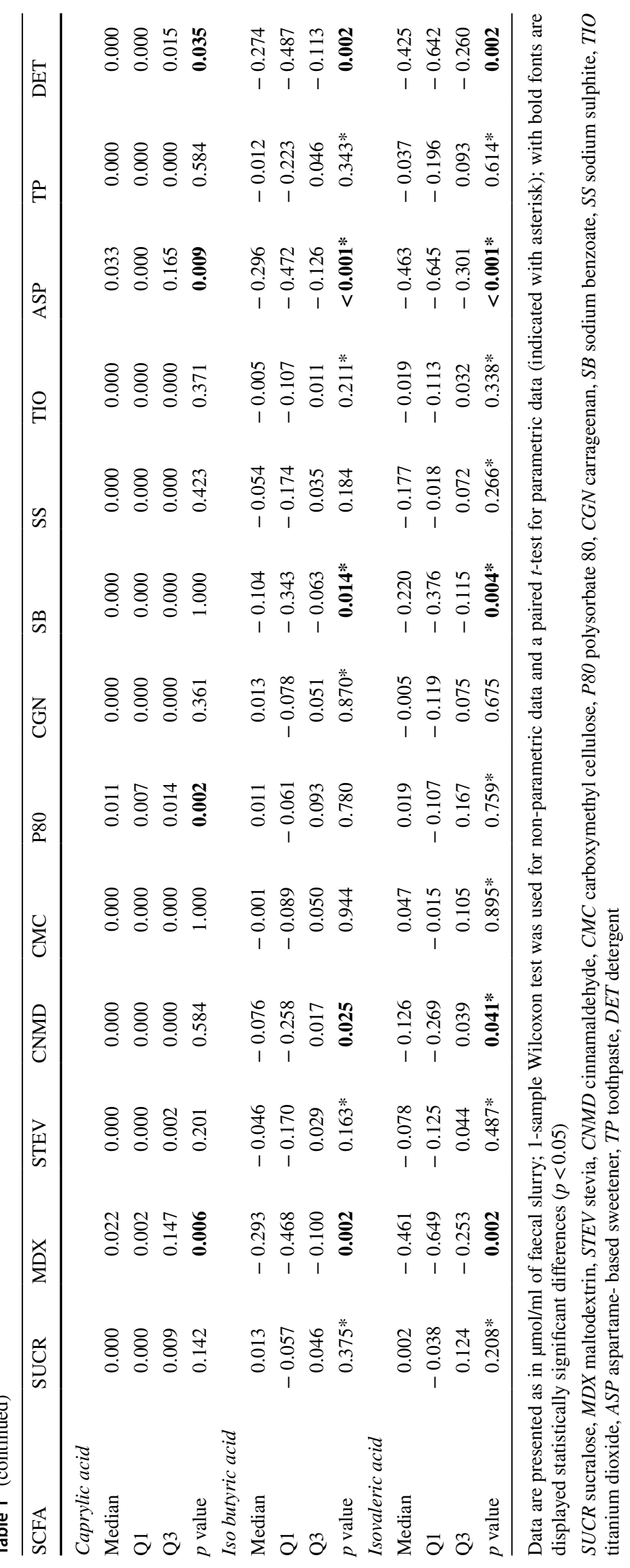



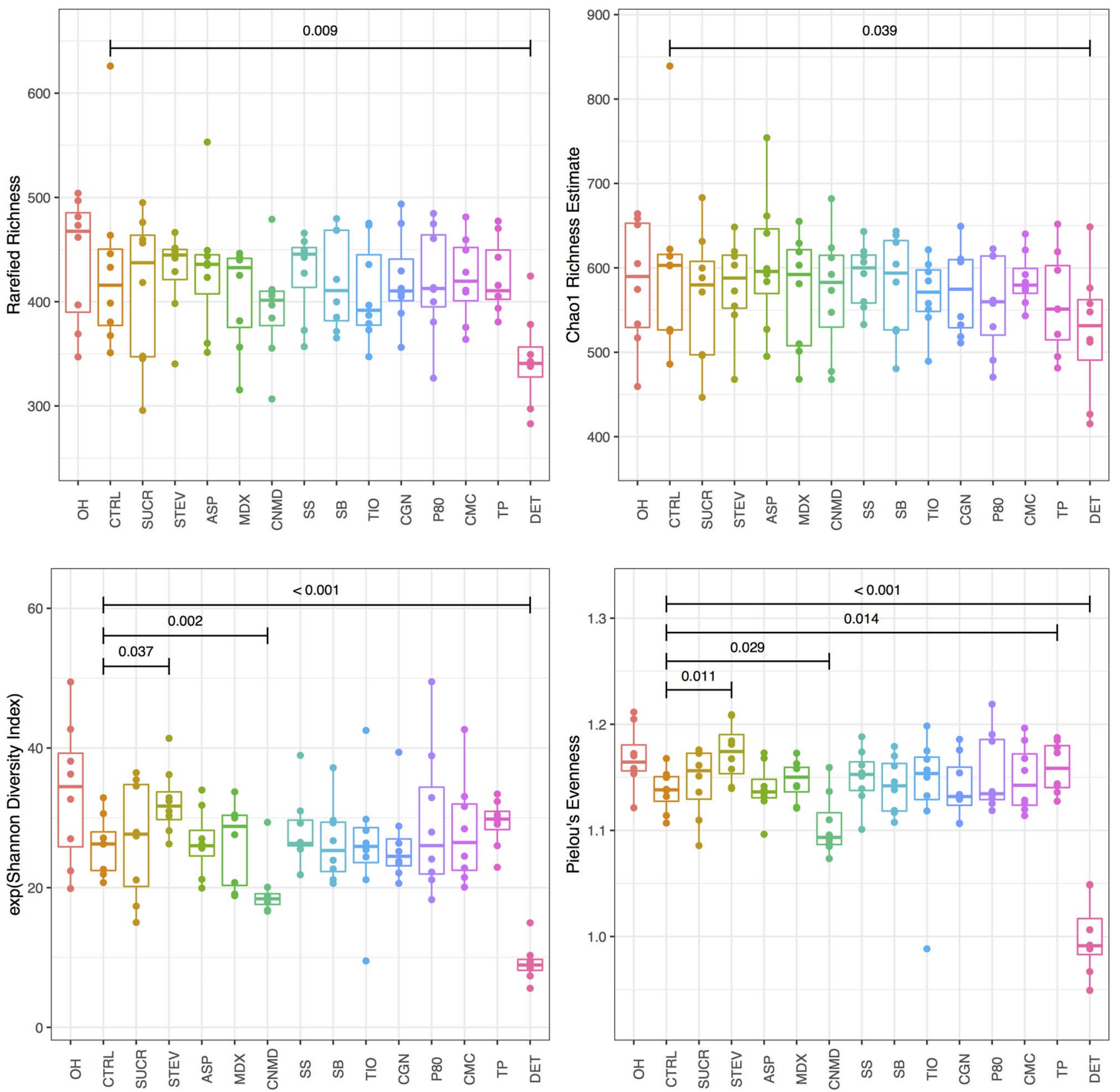

Fig. 2 Microbiome $\alpha$-diversity indices before and following $24 \mathrm{~h}$ batch faecal fermentation of fibre with food additives, artificial sweeteners and domestic hygiene products. $O H$ baseline, CTRL control, SUCR sucralose, STEV stevia, ASP aspartame based sweetener,

$\left(R^{2}=20.6 \%, p=0.016\right)$ and dishwashing detergent $\left(R^{2}=63.4 \%, p=0.008\right)$ (Fig. 4). The effect of dishwashing liquid and cinnamaldehyde on microbiome community structure dominated that of inter-subject variation (Figs. 3, 4).

\section{Effect of additives on taxon relative abundance}

In accordance with the significant shifts observed on $\alpha$ and $\beta$ diversity, major effects in taxon relative abundance were observed with the fermentation of fibre in the presence of 
Fig. 3 Microbiome community structure ( $\beta$ diversity) using the Bray-Curtis dissimilarity index before and following $24 \mathrm{~h}$ batch faecal fermentation of fibre with food additives, artificial sweeteners and domestic hygiene products. $O H$ baseline, CTRL control, SUCR sucralose, $S T E V$ stevia, $A S P$ aspartame based sweetener, $M D X$ maltodextrin, $C N M D$ cinnamaldehyde, $S S$ sodium sulphite, $S B$ sodium benzoate, $T I O$ titanium dioxide, $C G N$ carrageenan-kappa, $P 80$ polysorbate-80, CMC carboxymethyl cellulose, TP toothpaste, DET detergent

cinnamaldehyde and dishwashing detergent (Fig. 5). Addition of dishwashing detergent increased the relative abundance of OTU belonging to Escherichia/Shigella and Klebsiella and in parallel decreased the relative abundance of 33 other OTUs, the majority of which belonged to Firmicutes. A similar increase of an OTU of Escherichia/Shigella was observed for cinnamaldehyde whereas 9 other OTUs, including three of Faecalibacterium and four of Subdoligranulum, all important butyrate producers, significantly decreased (Fig. 5). The relative abundance of Escherichia/Shigella also increased in the presence of sucralose and carrageenankappa. Similarly, a species of Bilophila increased with the addition of sucralose, sodium sulphite and polysorbate- 80 . Except for dishwashing detergent and cinnamaldehyde, major declines in the abundance of OTUs of Faecalibacterium and Subdoligranulum were observed using polysorbate- 80 as substrate. There was no effect on the addition of maltodextrin, stevia, titanium dioxide and toothpaste on OTU relative abundance (Fig. 5). Similar effects were observed at genus and at the family level (Online Resource $4)$.

\section{Effect of additives on the growth of major bacterial groups}

Figure 6 shows the absolute concentration and Table 2 the median difference of $16 \mathrm{~S}$ rRNA gene copy number, between the various additives and the control, for each bacterial group tested. Regardless of the type of additive tested, the concentration of total bacteria significantly increased after $24 \mathrm{~h}$ fermentation and Bacteroides/Prevotella and C. leptum cluster typically represented the two most dominant groups (Fig. 6). Among the additives, the addition of carrageenankappa $(p=0.034)$ and dishwashing detergent $(p=0.002)$, significantly decreased the concentration of total bacteria in comparison with the control group (Fig. 6). Similarly, maltodextrin $(p=0.021)$ and sodium benzoate $(p<0.001)$ significantly decreased the concentration of $E$. coli whereas addition of cinnamaldehyde $(p=0.014)$, sodium sulphite $(p=0.038)$ or dishwashing detergent $(p<0.001)$ promoted their growth (Fig. 6). The growth of species belonging to C. leptum significantly decreased in the presence of cinnamaldehyde $(p=0.003)$, polysorbate-80 $(p=0.001)$, titanium dioxide $(p=0.029)$ and dishwashing detergent $(p<0.001)$
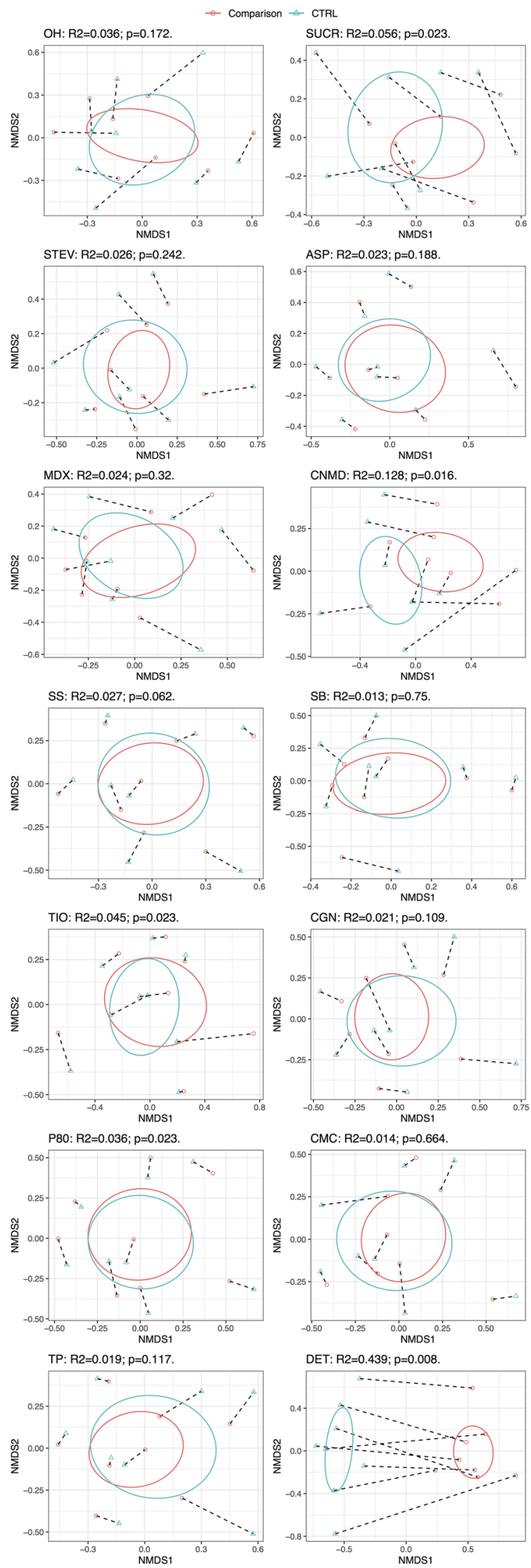
Fig. 4 Microbiome community structure ( $\beta$ diversity) using the UniFrac unweighted distances before and following $24 \mathrm{~h}$ batch faecal fermentation of fibre with food additives, artificial sweeteners and domestic hygiene products. $O H$ baseline, CTRL control, SUCR sucralose, $S T E V$ stevia, $A S P$ aspartame based sweetener, $M D X$ maltodextrin, $C N M D$ cinnamaldehyde, $S S$ sodium sulphite, $S B$ sodium benzoate, $T I O$ titanium dioxide, $C G N$ carrageenan-kappa, $P 80$ polysorbate-80, CMC carboxymethyl cellulose, $T P$ toothpaste, $D E T$ detergent

and it was also the case for Bacteroides/Prevotella, when aspartame-based sweetener $(p=0.048)$ or dishwashing detergent were added ( $p=0.001)$ (Fig. 6). Bifidobacterium growth increased from the control with the addition of maltodextrin $(p=0.002)$, sodium benzoate $(p=0.008)$ and aspartame-based sweetener $(p=0.005)$ (Fig. 6). In contrast, a significant inhibitory effect on Bifidobacterium was observed with polysorbate- $80(p=0.036)$, carrageenankappa $(p=0.003)$, sodium sulphite $(p=0.013)$ or dishwashing detergent $(p<0.001)$ (Fig. 6). When compared with the control group, cinnamaldehyde $(p=0.003)$, carrageenankappa $(p=0.014)$, sodium sulphite $(p=0.001)$ and dishwashing detergent $(p=0.014)$ significantly inhibited the growth of the $B$. coccoides group whereas maltodextrin $(p=0.002)$ and aspartame-based sweetener $(p=0.009)$ significantly promoted this (Fig. 6). Stevia, sucralose, carboxymethyl cellulose and toothpaste had no significant effects on the growth of these broad bacterial populations (Fig. 6).

\section{Discussion}

It has become increasingly accepted that a diverse gut microbiome with high production of SCFA, particularly butyric acid, is an independent biomarker of host health. It is also known that diet influences the gut microbiome structure and function, including its fibre fermentation capacity [32]. However, relatively little is known about what effect that food additives, artificial sweeteners and accidental exposure to domestic hygiene products might have on the gut microbiome. As our diet has become more industrialised and is expected to become even more so to sustain food availability, it is important to understand the beneficial or detrimental effect food additives may have on the gut microbiome, and by extension to host health, to guide current and future use.

This study measured the effect of thirteen commonly used food additives, artificial sweeteners, and domestic hygiene products on the healthy gut microbiome composition and its fermentation capacity using in-vitro human microbiome batch fermentations. Changes in the ability of the gut microbiome to ferment fibre and produce SCFA and quantitative changes in major bacterial groups were measured and the summary results of this study are presented in Fig. 7. In addition to these analyses, the global microbiome
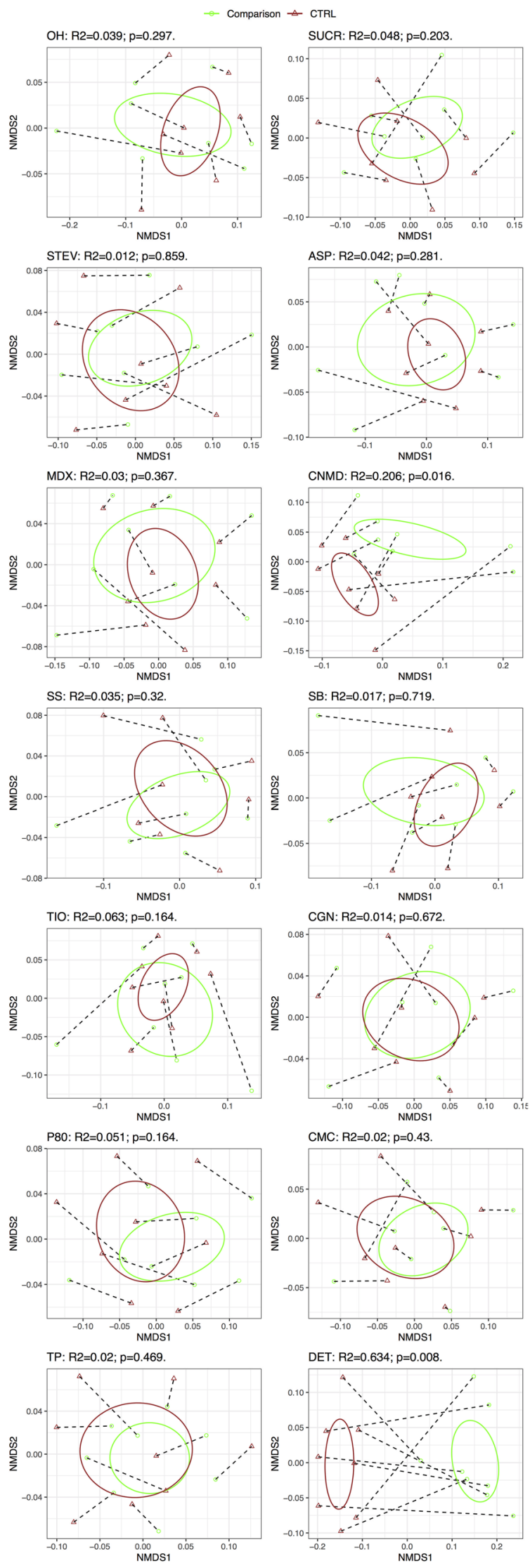
Fig. 5 The effect of food additives, artificial sweeteners and domestic hygiene products on bacterial OTU relative abundance. $O H$ baseline, $C T R L$ control, SUCR sucralose, STEV stevia, $A S P$ aspartame based sweetener, $M D X$ maltodextrin, $C N M D$ cinnamaldehyde, $S S$ sodium sulphite, $S B$ sodium benzoate, $T I O$ titanium dioxide, $C G N$ carrageenan-kappa, $P 80$ polysorbate-80, $C M C$ carboxymethyl cellulose, $T P$ toothpaste, $D E T$ detergent, $\log 2(F C) \log 2$ fold change

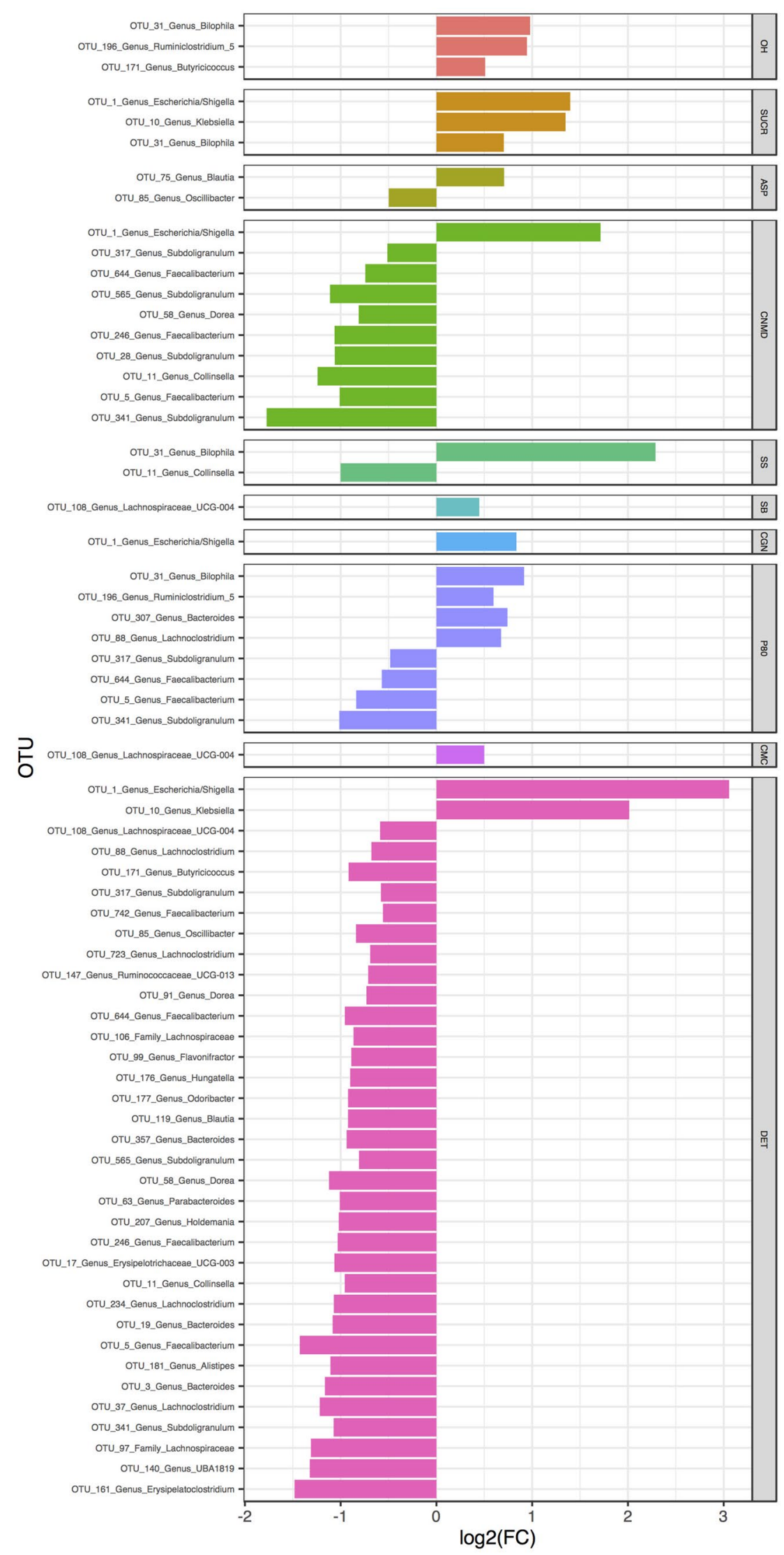



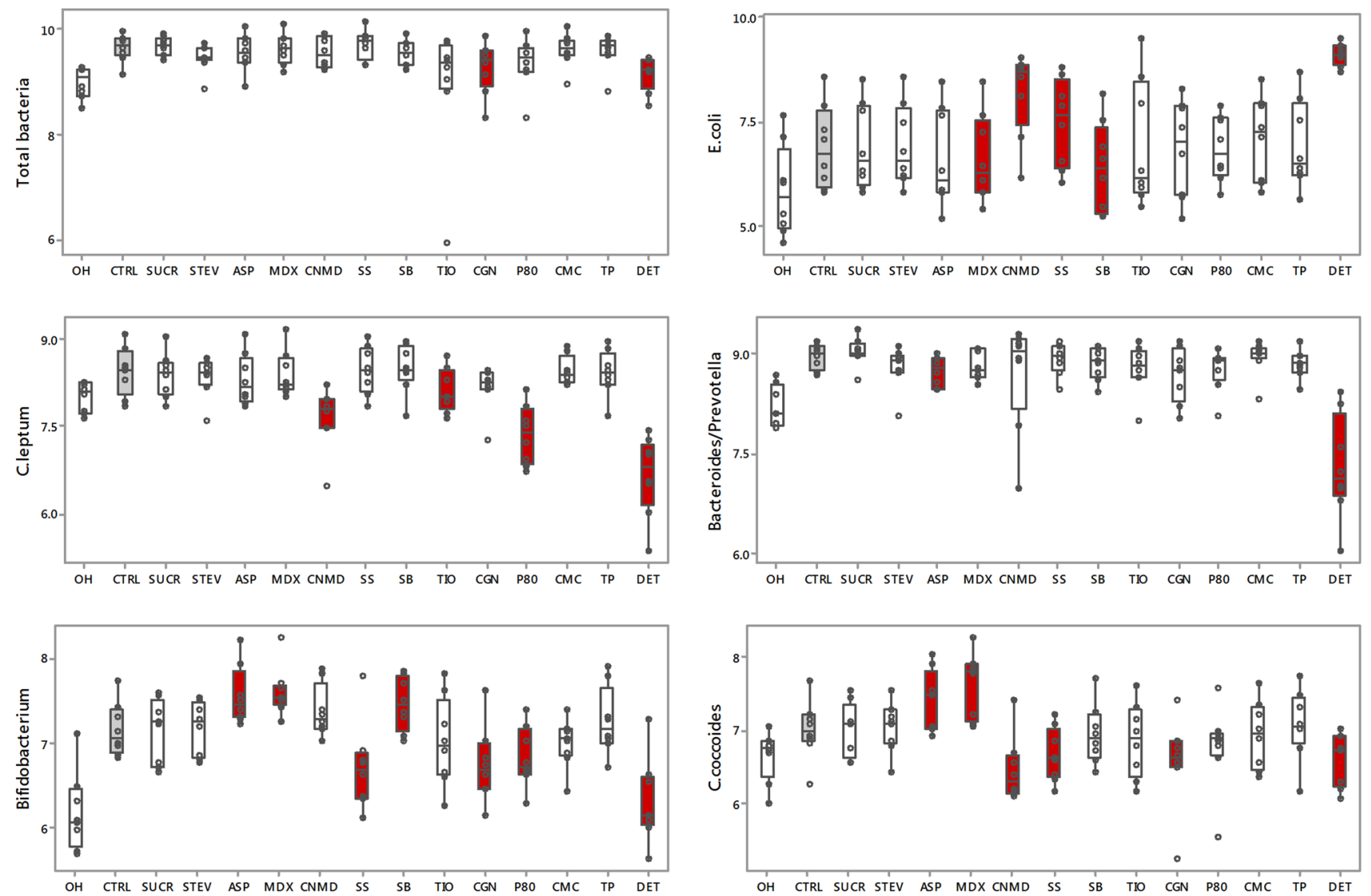

Fig. 6 Concentration of total and major bacterial groups (number of 16S rRNA gene copies/ml) before and following $24 \mathrm{~h}$ batch faecal fermentation of fibre with food additives, artificial sweeteners and domestic hygiene products. Red filling boxplot indicates significant difference $(p<0.05)$ compared with the CTRL (displayed with grey

composition and community structure were characterised using 16S rRNA gene amplicon sequencing as displayed in Fig. 8. Six of the additives affected the production of SCFA, five influenced the global microbiome community structure and nine altered the concentration of dominant microbial groups. Only toothpaste, stevia and carboxymethyl cellulose showed no or minimal effects on the broad composition and fermentation capacity of the faecal microbiome. However, for the additives for which an effect was observed, changes in microbiome composition and SCFA concentrations varied considerably among them; in terms of both the microorganisms or SCFA affected as well as the direction of this effect. Thus, this study highlights that the gut microbiome is modifiable in different ways by different additives. These variable effects of various food additives also suggest that their impact on the gut microbiome needs to be studied separately for each, in combination with each other, and in addition to other macronutrients, micronutrients and fibre in our diet.

There is increasing interest in the effect of the food industrialisation on human health and particularly on

filling boxplot); $O H$ baseline, CTRL control, SUCR sucralose, STEV stevia, $A S P$ aspartame based sweetener, $M D X$ maltodextrin, $C N M D$ cinnamaldehyde, $S S$ sodium sulphite, $S B$ sodium benzoate, $T I O$ titanium dioxide, $C G N$ carrageenan-kappa, $P 80$ polysorbate-80, $C M C$ carboxymethyl cellulose, $T P$ toothpaste, $D E T$ detergent

non-communicable disease, such as inflammatory bowel disease and diabetes $[10,20]$. In previous studies, these effects were associated directly or indirectly with the microbiome of the large bowel. A food additive can affect gut homeostasis by influencing either the gut microbiome, the mucus layer or both. Carrageenan-kappa, upon consumption, has been associated with an increased prevalence of intestinal lesions in animal models [33], highlighting a detrimental effect on the mucosal barrier. Recent evidence from experiments in mice shows that this effect may be mediated by changes in the abundance of Akkermansia muciniphila, a potent antiinflammatory bacterium. The results of the current study show that similar effects were observed with inhibition in the growth of Bifidobacterium and B. coccoides cluster, members of which have beneficial effects for the host [34]. Similarly, the dietary emulsifiers carboxymethyl cellulose and polysorbate- 80 have been proposed to directly alter human microbiome composition and ex-vivo gene expression, potentiating intestinal inflammation [21]. Although in our current study no effect of carboxymethyl cellulose 


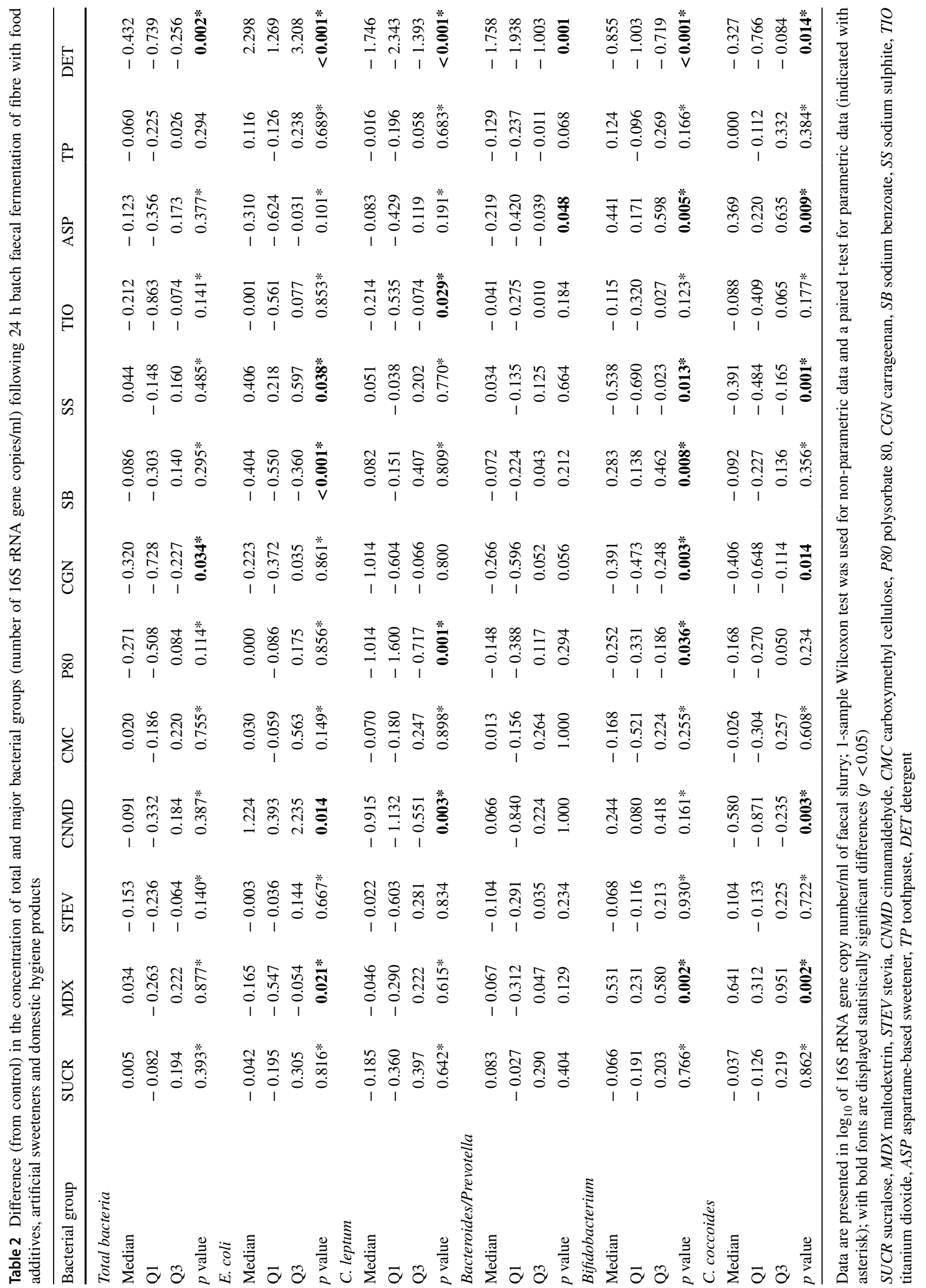


Fig. 7 Heatmap illustrating the summary effects of food additives, artificial sweeteners and domestic hygiene products on net production of total and individual short chain fatty acids and concentration of total and major bacterial groups. $O H$ baseline, $C T R L$ control, SUCR sucralose, STEV stevia, $A S P$ aspartame based sweetener, $M D X$ maltodextrin, $C N M D$ cinnamaldehyde, $S S$ sodium sulphite, $S B$ sodium benzoate, $T I O$ titanium dioxide, $C G N$ carrageenan-kappa, $P 80$ polysorbate- $80, C M C$ carboxymethyl cellulose, $T P$ toothpaste, DET detergent. Red indicates a decrease and green an increase in the concentration of short chain fatty acids or bacterial groups

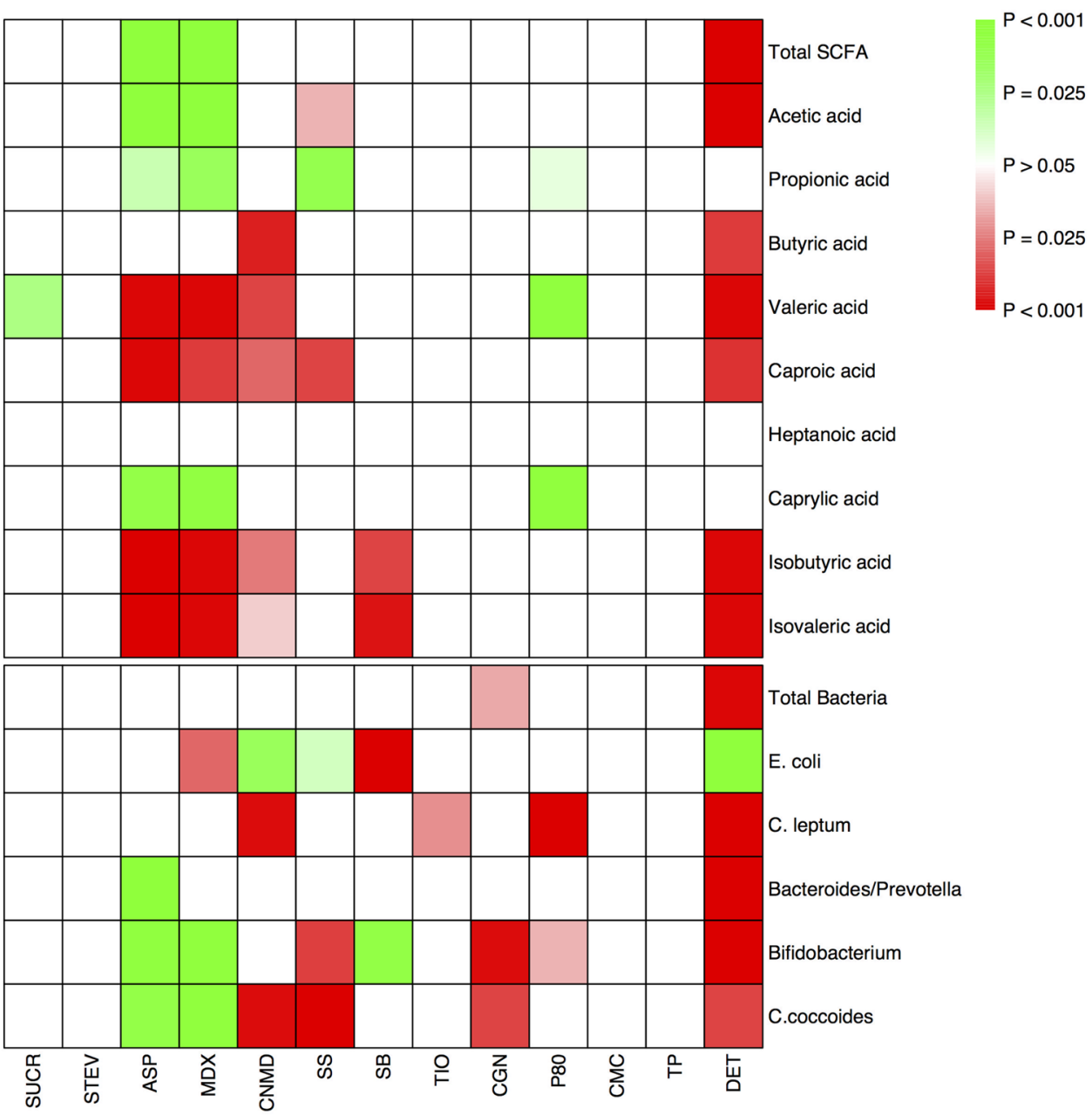

was seen on the fermentation capacity or on shifts in major bacterial groups, polysorbate- 80 decreased the growth of Bifidobacterium and C. leptum and the relative abundance of other Firmicutes as confirmed by the results of both qPCR and $16 \mathrm{~S}$ rRNA gene sequencing. Inorganic sulphite salts are frequently used to stop fermentation in wine and beer as well as antioxidants in food. This bacteriostatic effect of sodium sulphite was observed for members of the genus Bifidobacterium and the cluster $B$. coccoides and this effect may give a growth advantage to E. coli and Bilophila wadsworthia which gain energy through sulphite respiration [35]. Irwin et al. have previously described the bactericidal effects of sodium sulphite on probiotic-type bacteria, common members of the human gut microbiome [36]. The exact opposite effects were observed for the growth of Bifidobacterium, the cluster $B$. coccoides and $E$. coli when either maltodextrin or the aspartame-based sweetener was present. This is likely to be because maltodextrin is an artificially produced glucose polymer which, if not absorbed in the small intestine, has prebiotic properties in the colon [37]. Therefore, the increase in the probiotic genus Bifidobacterium and B. coccoides and the corresponding decrease in $E$. coli is most likely due to the fact that the former two use maltodextrin for growth [38, 39] instigating fermentation, production of acetic acid and creating an acidic environment in which $E$. coli growth is suppressed. Interestingly, changes in the absolute concentration of these three dominant bacterial groups, quantified with qPCR, were not in parallel the absence of effects observed using next generation sequencing. Sodium benzoate has been shown to decrease plasma ammonium levels by reducing glycine metabolism to treat patients with urea-cycle-disorder and acute hyperammonaemia [40, 41]. Use of sodium benzoate in this study increased the beneficial Bifidobacterium but reduced $E$. coli and the concentration of BCFA, suggesting that protein fermentation and potentially production of ammonia from bacterial metabolism in the gut is diminished. Similar to maltodextrin, these effects were not observed with in-depth characterisation of the microbiome using $16 \mathrm{~S}$ rRNA sequencing. However, discordant results are to be expected as qPCR provides an absolute quantification of broader groups of bacteria and $16 \mathrm{~S}$ rRNA sequencing offers proportional representation of the overall microbial community. The aspartame-based sweetener we used in this study was rich in maltodextrin in addition to, aspartame and 
Fig. 8 Heatmap illustrating the effects of food additives, artificial sweeteners and domestic hygiene products on mean relative abundance of the top 50 dominant bacterial OTUs across all samples. $O H$ baseline, $C T R L$ control, SUCR sucralose, STEV stevia, $A S P$ aspartame based sweetener, $M D X$ maltodextrin, $C N M D$ cinnamaldehyde, $S S$ sodium sulphite, $S B$ sodium benzoate, $T I O$ titanium dioxide, $C G N$ carrageenan-kappa, $P 80$ polysorbate-80, CMC carboxymethyl cellulose, $T P$ toothpaste, DET detergent, OTU Operational Taxonomic Unit

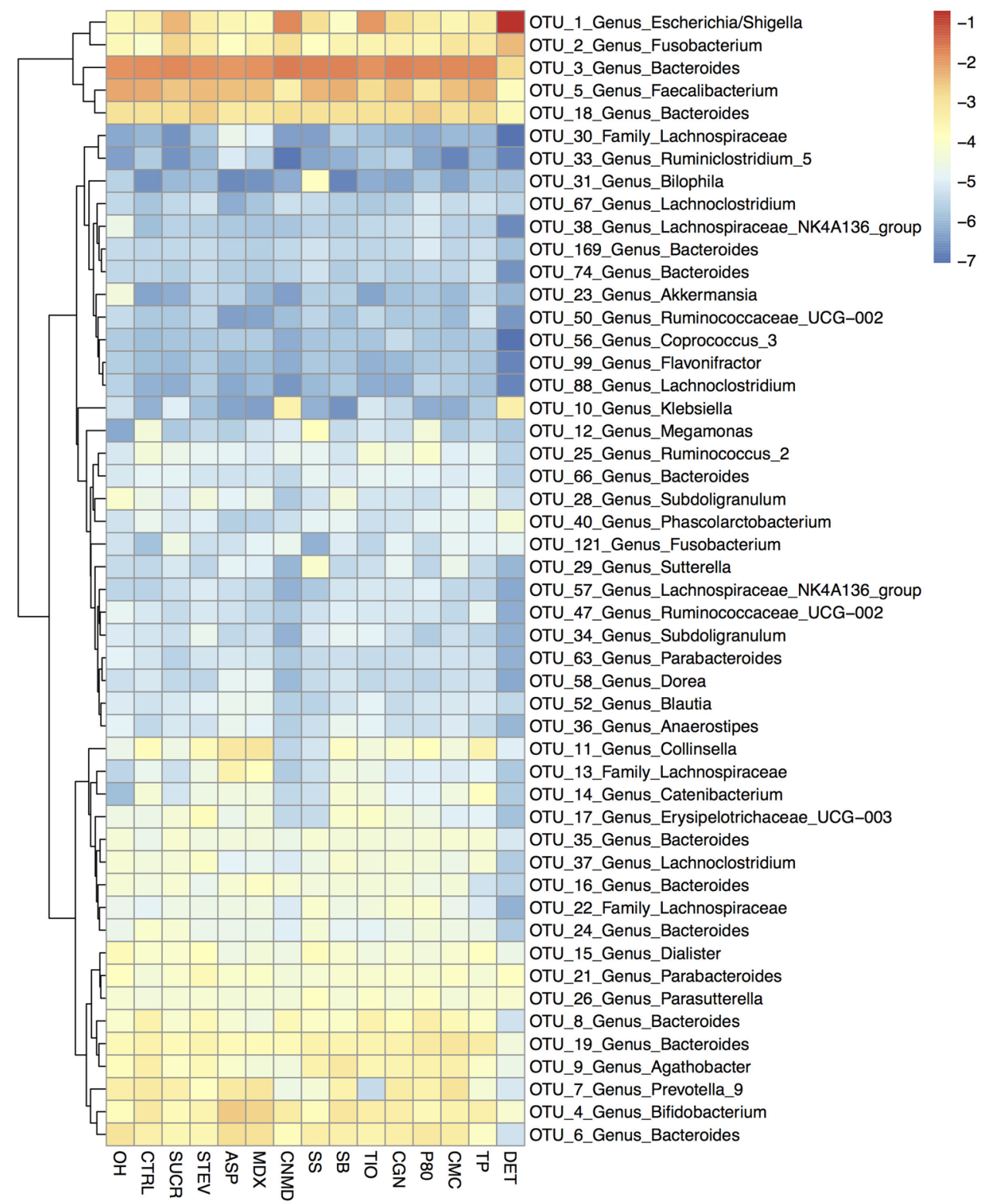

acesulflame potassium. This, therefore, prevented the study of aspartame in isolation. However, the absence of major differences between the maltodextrin and the aspartame-based sweetener suggests that most of the effect seen on the gut microbiome comes from maltodextrin with no major contributions of aspartame and acesulfame potassium; at least in the amount we tested in this experiment which equals $8 \%$ of the estimated daily intake which might carry-over to the gut.

Crohn's disease has been characterised by a gut microbiome with a reduced number of Firmicutes, such as species belonging to $\mathrm{C}$. leptum, Bifidobacterium and Bacteroidetes and an increase in Proteobacteria, particularly E. coli strains with adherent and invasive properties [22, 24]. Interestingly, the addition of cinnamaldehyde, a cinnamon ingredient, or dishwashing detergent increased the E. coli and decreased the $C$. leptum and B. coccoides growth. A similar effect was also observed for polysorbate- 80 with a diminished abundance of butyrate-producing species and increase in a species of Bilophila, a hydrogen sulphide producer implicated in colitis in IL-10 knockout mice [42]. Assuming that the gut microbial dysbiosis seen in patients with Crohn's disease is a primary defect of the disease, and such species are implicated in disease pathogenesis, these findings suggest that consumption of cinnamon-containing food, polysorbate- 80 and accidental ingestion of residual detergent on crockery and utensils may exacerbate dysbiosis and influence disease outcomes. Dishwashing detergent contains surfactants, which lower the surface tension, potentially causing degradation of mucus layer and the mucosal barrier to break-down thus potentially affecting microbial composition 
[43] particularly microbes which are adjacent to epithelial cells. Many Firmicutes like Faecalibacterium and Subdoligranulum are butyric acid-producing bacteria; hence the diminishing production of butyric acid here coincides with the decline in the concentration and abundance of these taxa with the addition of cinnamaldehyde, and dishwashing detergent. Firmicutes constitute a large proportion of the bacteria in the human gut microbiome, therefore, a significant change to the composition and functionality found within this phylum could, in theory, have detrimental consequences to the host. Butyric acid, for example, is the preferable energy substrate for the colonocytes and regulates regulatory $\mathrm{T}$ cells which play an important role in cell-mediated immunity [44]. A similar effect on C. leptum was seen for polysorbate- 80 and a modest one for titanium dioxide. Collectively this evidence proposes that these additives could exacerbate the microbial dysbiosis seen in inflammatory bowel disease.

This study looked at the effect of food additives, artificial sweeteners and domestic hygiene products on the gut microbiome composition and fibre fermentation capacity in healthy human individuals, using batch fermentations with human faecal inoculum; thus, complementing previous research in animals. Although in the current study the SCFA and microbiome composition profile of the control, following $24 \mathrm{~h}$ fermentation, are in accordance to those that occur in the human gut, batch fermentation is a snapshot and not an exact simulant of human gut physiology and its complex dynamics $[45,46]$. This may explain some of the discrepancies between the findings of this study and previous research [10]. Batch faecal fermentations do, however, provide crucial preclinical data, under well-controlled experimental conditions. They enable exploration of various additives at the same time and the direct effect on the gut microbiome in isolation of the host effect; hence bridging the gap between animal research and human trials. The data generated from this study offer important insights on where future research on additives should be directed, using animal experiments and human randomised controlled trials. In our case, this may be relevant for cinnamaldehyde, polysorbate-80, sodium sulphite, sodium benzoate, sucralose and dishwashing detergent but not for carboxymethyl cellulose, and stevia. While maltodextrin and the aspartame-based sweetener influenced the gut microbiome composition and production of SCFA, they did not induce dysbiosis and their effect might be considered favourable by inhibiting the growth of $E$. coli, thus promoting Bifidobacterium and correspondingly increasing the production of acetic acid and propionic acid. This bifidogenic effect of maltodextrin, an artificial glucose polymer has been observed previously too [47]. These findings are in contrast to evidence suggesting that maltodextrin induces dysbiosis promoting gut inflammation [10]. Such discrepancies might be explained by broad differences in the methodology applied among studies and the fact that in the current study we explored the effect of maltodextrin on the gut microbiome in isolation of the host and gut physiology. However, maltodextrin is the main source of carbohydrate in proprietary feeds used for the amelioration of gut inflammation with exclusive enteral nutrition in active Crohn's disease $[48,49]$. This reproducible clinical evidence challenges our current perceptions on the role of maltodextrin on gut inflammation.

This study contributes to the limited knowledge on the effect of food additives, artificial sweeteners and domestic hygiene products on the human gut microbiome composition and fibre fermentation capacity. We have shown that the presence of certain additives changed the microbial composition, and this became similar to the gut microbiome seen in individuals with either inflammatory bowel disease or obesity. For other additives, their effects were counterintuitive and opposite to animal research, implicating them in gut inflammation, and by proxy to human inflammatory bowel disease $[10,50]$. This study underpins the importance of evaluating each additive separately and not grouped by their functional class. Here, we lay the groundwork for future research into individual additives on the gut microbiome composition and its fermentation capacity measured over a longer time period both in public health research and in the context of therapeutic interventions in patients with established dysbiosis, including patients with inflammatory bowel disease.

Funding Dr Ben Nichols was funded by a grant from the Biotechnology and Biological Sciences Research Council (BB/R006539/1).

\section{Compliance with ethical standards}

Conflict of interests The authors have no conflicts of interest to disclose.

Open Access This article is licensed under a Creative Commons Attribution 4.0 International License, which permits use, sharing, adaptation, distribution and reproduction in any medium or format, as long as you give appropriate credit to the original author(s) and the source, provide a link to the Creative Commons licence, and indicate if changes were made. The images or other third party material in this article are included in the article's Creative Commons licence, unless indicated otherwise in a credit line to the material. If material is not included in the article's Creative Commons licence and your intended use is not permitted by statutory regulation or exceeds the permitted use, you will need to obtain permission directly from the copyright holder. To view a copy of this licence, visit http://creativecommons.org/licenses/by/4.0/.

\section{References}

1. El Kaoutari A, Armougom F, Gordon JI, Raoult D, Henrissat B (2013) The abundance and variety of carbohydrate-active enzymes in the human gut microbiota. Nat Rev Microbiol 11(7):497-504. https://doi.org/10.1038/nrmicro3050 
2. Larsbrink J, Rogers TE, Hemsworth GR, McKee LS, Tauzin AS, Spadiut O, Klinter S, Pudlo NA, Urs K, Koropatkin NM, Creagh AL, Haynes CA, Kelly AG, Cederholm SN, Davies GJ, Martens EC, Brumer H (2014) A discrete genetic locus confers xyloglucan metabolism in select human gut Bacteroidetes. Nature 506(7489):498-502. https://doi.org/10.1038/nature12907

3. den Besten G, van Eunen K, Groen AK, Venema K, Reijngoud DJ, Bakker BM (2013) The role of short-chain fatty acids in the interplay between diet, gut microbiota, and host energy metabolism. J Lipid Res 54(9):2325-2340. https://doi.org/10.1194/jlr.R036012

4. O'Callaghan A, van Sinderen D (2016) Bifidobacteria and Their Role as Members of the Human Gut Microbiota. Frontiers in microbiology 7:925. https://doi.org/10.3389/fmicb.2016.00925

5. Ley RE, Backhed F, Turnbaugh P, Lozupone CA, Knight RD, Gordon JI (2005) Obesity alters gut microbial ecology. Proc Natl Acad Sci USA 102(31):11070-11075. https://doi.org/10.1073/ pnas.0504978102

6. Puddu A, Sanguineti R, Montecucco F, Viviani GL (2014) Evidence for the gut microbiota short-chain fatty acids as key pathophysiological molecules improving diabetes. Mediators Inflamm 2014:162021. https://doi.org/10.1155/2014/162021

7. Manichanh C, Rigottier-Gois L, Bonnaud E, Gloux K, Pelletier E, Frangeul L, Nalin R, Jarrin C, Chardon P, Marteau P, Roca J, Dore J (2006) Reduced diversity of faecal microbiota in Crohn's disease revealed by a metagenomic approach. Gut 55(2):205-211. https://doi.org/10.1136/gut.2005.073817

8. Lambeth SM, Carson T, Lowe J, Ramaraj T, Leff JW, Luo L, Bell CJ, Shah VO (2015) Composition, diversity and abundance of gut microbiome in prediabetes and type 2 diabetes. J Diabetes Obes 2(3):1-7. https://doi.org/10.15436/2376-0949.15.031

9. De Filippis F, Pellegrini N, Vannini L, Jeffery IB, La Storia A, Laghi L, Serrazanetti DI, Di Cagno R, Ferrocino I, Lazzi C, Turroni S, Cocolin L, Brigidi P, Neviani E, Gobbetti M, O'Toole PW, Ercolini D (2016) High-level adherence to a Mediterranean diet beneficially impacts the gut microbiota and associated metabolome. Gut 65(11):1812-1821. https://doi.org/10.1136/gutjn 1-2015-309957

10. Levine A, Sigall Boneh R, Wine E (2018) Evolving role of diet in the pathogenesis and treatment of inflammatory bowel diseases. Gut 67(9):1726-1738. https://doi.org/10.1136/gutjnl-2017-31586 6

11. Monteiro CA, Moubarac JC, Levy RB, Canella DS, Louzada M, Cannon G (2018) Household availability of ultra-processed foods and obesity in nineteen European countries. Public Health Nutr 21(1):18-26. https://doi.org/10.1017/s1368980017001379

12. Zinocker MK, Lindseth IA (2018) The Western diet-Microbiomehost interaction and its role in metabolic disease. Nutrients 10(3). doi: 10.3390/nu10030365

13. Marion-Letellier R, Amamou A, Savoye G, Ghosh S (2019) Inflammatory bowel diseases and food additives: to add fuel on the flames! Nutrients 11(5). doi: 10.3390/nu11051111

14. Rodriguez-Palacios A, Harding A, Menghini P, Himmelman C, Retuerto M, Nickerson KP, Lam M, Croniger CM, McLean MH, Durum SK, Pizarro TT, Ghannoum MA, Ilic S, McDonald C, Cominelli F (2018) The Artificial sweetener splenda promotes gut proteobacteria, dysbiosis, and myeloperoxidase reactivity in Crohn's disease-like Ileitis. Inflamm Bowel Dis 24(5):1005-1020. https://doi.org/10.1093/ibd/izy060

15. Abou-Donia MB, El-Masry EM, Abdel-Rahman AA, McLendon RE, Schiffman SS (2008) Splenda alters gut microflora and increases intestinal p-glycoprotein and cytochrome p-450 in male rats. J Toxicol Environ Health Part A 71(21):1415-1429. https:// doi.org/10.1080/15287390802328630

16. Suez J, Korem T, Zeevi D, Zilberman-Schapira G, Thaiss CA, Maza O, Israeli D, Zmora N, Gilad S, Weinberger A, Kuperman Y, Harmelin A, Kolodkin-Gal I, Shapiro H, Halpern Z, Segal E,
Elinav E (2014) Artificial sweeteners induce glucose intolerance by altering the gut microbiota. Nature 514(7521):181-186. https ://doi.org/10.1038/nature13793

17. Rhodes JM (2018) Dietary exposure to emulsifiers and detergents and the prevalence of cardiovascular disease. QJM 111(5):283286. https://doi.org/10.1093/qjmed/hcx087

18. Cummings JH, Bingham SA, Heaton KW, Eastwood MA (1992) Fecal weight, colon cancer risk, and dietary intake of nonstarch polysaccharides (dietary fiber). Gastroenterology 103(6):17831789. https://doi.org/10.1016/0016-5085(92)91435-7

19. Gibson R, Eriksen R, Chambers E, Gao H, Aresu M, Heard A, Chan Q, Elliott P, Frost G (2019) Intakes and food sources of dietary fibre and their associations with measures of body composition and inflammation in UK adults: cross-sectional analysis of the airwave health monitoring study. Nutrients 11(8):1839

20. Chassaing B, Koren O, Goodrich JK, Poole AC, Srinivasan S, Ley RE, Gewirtz AT (2015) Dietary emulsifiers impact the mouse gut microbiota promoting colitis and metabolic syndrome. Nature 519(7541):92-96. https://doi.org/10.1038/nature14232

21. Chassaing B, Van de Wiele T, De Bodt J, Marzorati M, Gewirtz AT (2017) Dietary emulsifiers directly alter human microbiota composition and gene expression ex vivo potentiating intestinal inflammation. Gut 66(8):1414-1427. https://doi.org/10.1136/gutjn 1-2016-313099

22. Gerasimidis K, Bertz M, Hanske L, Junick J, Biskou O, Aguilera M, Garrick V, Russell RK, Blaut M, McGrogan P, Edwards CA (2014) Decline in presumptively protective gut bacterial species and metabolites are paradoxically associated with disease improvement in pediatric Crohn's disease during enteral nutrition. Inflamm Bowel Dis 20(5):861-871. https://doi.org/10.1097/ MIB.0000000000000023

23. Svolos V, Hansen R, Nichols B, Quince C, Ijaz UZ, Papadopoulou RT, Edwards CA, Watson D, Alghamdi A, Brejnrod A, Ansalone C, Duncan H, Gervais L, Tayler R, Salmond J, Bolognini D, Klopfleisch R, Gaya DR, Milling S, Russell RK, Gerasimidis K (2019) Treatment of active Crohn's disease with an ordinary food-based diet that replicates exclusive enteral nutrition. Gastroenterology 156(5):1354-1367 e1356. doi: 10.1053/j.gastro.2018.12.002

24. Quince C, Ijaz UZ, Loman N, Eren AM, Saulnier D, Russell J, Haig SJ, Calus ST, Quick J, Barclay A, Bertz M, Blaut M, Hansen R, McGrogan P, Russell RK, Edwards CA, Gerasimidis K (2015) Extensive modulation of the fecal metagenome in children with Crohn's disease during exclusive enteral nutrition. Am J Gastroenterol 110(12):1718-1729; quiz 1730. doi: 10.1038/ajg.2015.357

25. Rognes T, Flouri T, Nichols B, Quince C, Mahe F (2016) VSEARCH: a versatile open source tool for metagenomics. PeerJ 4:e2584. https://doi.org/10.7717/peerj.2584

26. Haas BJ, Gevers D, Earl AM, Feldgarden M, Ward DV, Giannoukos G, Ciulla D, Tabbaa D, Highlander SK, Sodergren E, Methe B, DeSantis TZ, Petrosino JF, Knight R, Birren BW (2011) Chimeric 16S rRNA sequence formation and detection in Sanger and 454-pyrosequenced PCR amplicons. Genome Res 21(3):494504. https://doi.org/10.1101/gr.112730.110

27. Edgar RC, Haas BJ, Clemente JC, Quince C, Knight R (2011) UCHIME improves sensitivity and speed of chimera detection. Bioinformatics 27(16):2194-2200. https://doi.org/10.1093/bioin formatics/btr381

28. Callahan BJ, McMurdie PJ, Rosen MJ, Han AW, Johnson AJ, Holmes SP (2016) DADA2: High-resolution sample inference from Illumina amplicon data. Nat Methods 13(7):581-583. https://doi. org/10.1038/nmeth.3869

29. Jari Oksanen FGB, Michael F, Roeland K, Pierre L, Dan M, Peter RM, O'Hara RB, Simpson GL, Solymos P, Henry M, Stevens H, Eduard S, Helene W (2019) Vegan: community ecology package. 
30. Price MN, Dehal PS, Arkin AP (2010) FastTree 2-approximately maximum-likelihood trees for large alignments. PLoS ONE 5(3):e9490. https://doi.org/10.1371/journal.pone.0009490

31. McMurdie PJ, Holmes S (2013) phyloseq: an R package for reproducible interactive analysis and graphics of microbiome census data. PLoS ONE 8(4):e61217. https://doi.org/10.1371/journ al.pone. 0061217

32. David LA, Maurice CF, Carmody RN, Gootenberg DB, Button JE, Wolfe BE, Ling AV, Devlin AS, Varma Y, Fischbach MA, Biddinger SB, Dutton RJ, Turnbaugh PJ (2014) Diet rapidly and reproducibly alters the human gut microbiome. Nature 505(7484):559-563. https://doi.org/10.1038/nature12820

33. Tobacman JK (2001) Review of harmful gastrointestinal effects of carrageenan in animal experiments. Environ Health Perspect 109(10):983-994. https://doi.org/10.1289/ehp.01109983

34. Shang Q, Sun W, Shan X, Jiang H, Cai C, Hao J, Li G, Yu G (2017) Carrageenan-induced colitis is associated with decreased population of anti-inflammatory bacterium, Akkermansia muciniphila, in the gut microbiota of C57BL/6J mice. Toxicol Lett 279:87-95. https://doi.org/10.1016/j.toxlet.2017.07.904

35. Carbonero F, Benefiel AC, Alizadeh-Ghamsari AH, Gaskins HR (2012) Microbial pathways in colonic sulfur metabolism and links with health and disease. Front Physiol 3:448. https://doi. org/10.3389/fphys.2012.00448

36. Irwin SV, Fisher P, Graham E, Malek A, Robidoux A (2017) Sulfites inhibit the growth of four species of beneficial gut bacteria at concentrations regarded as safe for food. PLoS ONE 12(10):e0186629. https://doi.org/10.1371/journal.pone.0186629

37. Patel S, Goyal A (2012) The current trends and future perspectives of prebiotics research: a review. 3 Biotech 2 (2):115-125. doi: 10.1007/s13205-012-0044-x

38. Pokusaeva K, Fitzgerald GF, van Sinderen D (2011) Carbohydrate metabolism in Bifidobacteria. Genes Nutr 6(3):285-306. https:// doi.org/10.1007/s12263-010-0206-6

39. Nickerson KP, Chanin R, McDonald C (2015) Deregulation of intestinal anti-microbial defense by the dietary additive, maltodextrin. Gut Microb 6(1):78-83. https://doi.org/10.1080/19490 976.2015.1005477

40. Enns GM, Berry SA, Berry GT, Rhead WJ, Brusilow SW, Hamosh A (2007) Survival after treatment with phenylacetate and benzoate for urea-cycle disorders. N Engl J Med 356(22):2282-2292. https ://doi.org/10.1056/NEJMoa066596

41. Liu J, Lkhagva E, Chung HJ, Kim HJ, Hong ST (2018) The pharmabiotic approach to treat hyperammonemia. Nutrients 10 (2). doi: 10.3390/nu10020140
42. Devkota S, Wang Y, Musch MW, Leone V, Fehlner-Peach H, Nadimpalli A, Antonopoulos DA, Jabri B, Chang EB (2012) Dietary-fat-induced taurocholic acid promotes pathobiont expansion and colitis in Il10-/- mice. Nature 487(7405):104-108. https://doi. org/10.1038/nature11225

43. Fathi-Azarbayjani A, Jouyban A (2015) Surface tension in human pathophysiology and its application as a medical diagnostic tool. BioImpacts 5(1):29-44. https://doi.org/10.15171/bi.2015.06

44. Canani RB, Costanzo MD, Leone L, Pedata M, Meli R, Calignano A (2011) Potential beneficial effects of butyrate in intestinal and extraintestinal diseases. World J Gastroenterol 17(12):1519-1528. https://doi.org/10.3748/wjg.v17.i12.1519

45. Williams CF, Walton GE, Jiang L, Plummer S, Garaiova I, Gibson GR (2015) Comparative analysis of intestinal tract models. Ann Rev Food Sci Technol 6:329-350. https://doi.org/10.1146/annur ev-food-022814-015429

46. Morrison DJ, Preston T (2016) Formation of short chain fatty acids by the gut microbiota and their impact on human metabolism. Gut Microb 7(3):189-200. https://doi.org/10.1080/19490 976.2015.1134082

47. Miyazato S, Kishimoto Y, Takahashi K, Kaminogawa S, Hosono A (2016) Continuous intake of resistant maltodextrin enhanced intestinal immune response through changes in the intestinal environment in mice. Biosci Microbiota Food Health 35(1):1-7. https ://doi.org/10.12938/bmfh.2015-009

48. Gerasimidis K, Nikolaou CK, Edwards CA, McGrogan P (2011) Serial fecal calprotectin changes in children with Crohn's disease on treatment with exclusive enteral nutrition: associations with disease activity, treatment response, and prediction of a clinical relapse. J Clin Gastroenterol 45(3):234-239. https://doi. org/10.1097/MCG.0b013e3181f39af5

49. Logan M, Clark CM, Ijaz UZ, Gervais L, Duncan H, Garrick V, Curtis L, Buchanan E, Cardigan T, Armstrong L, Delahunty C, Flynn DM, Barclay AR, Tayler R, McDonald E, Milling S, Hansen RK, Gerasimidis K, Russell RK (2019) The reduction of faecal calprotectin during exclusive enteral nutrition is lost rapidly after food re-introduction. Aliment Pharmacol Ther 50(6):664674. https://doi.org/10.1111/apt.15425

50. Khan MJ, Gerasimidis K, Edwards CA, Shaikh MG (2016) Role of Gut microbiota in the aetiology of obesity: proposed mechanisms and review of the literature. J Obes 2016:7353642. https:// doi.org/10.1155/2016/7353642

\section{Affiliations}

\section{Konstantinos Gerasimidis ${ }^{1}$ (1) - Katie Bryden ${ }^{1} \cdot$ Xiufen Chen $^{1}$ - Eleftheria Papachristou ${ }^{1}$. Anais Verney ${ }^{1}$. Marine Roig ${ }^{1} \cdot$ Richard Hansen $^{2} \cdot$ Ben Nichols ${ }^{1} \cdot$ Rodanthi Papadopoulou $^{1} \cdot$ Alison Parrett $^{1}$}

1 Human Nutrition, School of Medicine, Dentistry and Nursing, College of Medical, Veterinary and Life Sciences, University of Glasgow, New Lister Building, Glasgow Royal Infirmary, Glasgow G31 2ER, UK
2 Paediatric Gastroenterology, Hepatology and Nutrition, Royal Hospital for Children, 1345 Govan Road, Glasgow G51 4TF, UK 